\title{
Application of the direct Liapunov method to the problem of symmetric stability in the atmosphere
}

Article

Published Version

Cho, H.-R., Shepherd, T. G. and Vladimirov, V. A. (1993) Application of the direct Liapunov method to the problem of symmetric stability in the atmosphere. Journal of the Atmospheric Sciences, 50 (6). pp. 822-836. ISSN 1520-0469 doi: https://doi.org/10.1175/15200469(1993)050<0822:AOTDLM>2.0.CO;2 Available at https://centaur.reading.ac.uk/32917/

It is advisable to refer to the publisher's version if you intend to cite from the work. See Guidance on citing.

Published version at: http://dx.doi.org/10.1175/1520-0469(1993)050<0822:AOTDLM>2.0.CO;2

To link to this article DOI: http://dx.doi.org/10.1175/1520-

0469(1993)050<0822:AOTDLM>2.0.CO;2

Publisher: American Meteorological Society

All outputs in CentAUR are protected by Intellectual Property Rights law, including copyright law. Copyright and IPR is retained by the creators or other copyright holders. Terms and conditions for use of this material are defined in the End User Agreement. 


\section{CentAUR}

Central Archive at the University of Reading

Reading's research outputs online 


\title{
Application of the Direct Liapunov Method to the Problem of Symmetric Stability in the Atmosphere
}

\author{
H.-R. CHO AND T. G. SHEPHERD \\ Department of Physics, University of Toronto, Toronto, Ontario, Canada \\ V. A. Vladimirov \\ Lavrentyev Institute of Hydrodynamics, Novosibirsk, Russia \\ (Manuscript received 16 October 1991, in final form 22 April 1992)
}

\begin{abstract}
The problem of symmetric stability is examined within the context of the direct Liapunov method. The sufficient conditions for stability derived by Fjørtoft are shown to imply finite-amplitude, normed stability. This finite-amplitude stability theorem is then used to obtain rigorous upper bounds on the saturation amplitude of disturbances to symmetrically unstable flows.

By employing a virial functional, the necessary conditions for instability implied by the stability theorem are shown to be in fact sufficient for instability. The results of Ooyama are improved upon insofar as a tight twosided (upper and lower) estimate is obtained of the growth rate of (modal or nonmodal) symmetric instabilities.

The case of moist adiabatic systems is also considered.
\end{abstract}

\section{Introduction}

Studies of the problem of hydrodynamical stability may be divided into two general classes. The first is based on an explicit solution of the equations of motion, the spectral or normal-mode method being the most celebrated such example. The second class, in contrast, avoids a detailed consideration of the equations of motion and proceeds by the construction of some functional of the total problem. An important subset of this class is what is often referred to as the direct Liapunov method, following the pioneering work by Liapunov in 1892 on finite-dimensional mechanical systems.

In the past 10 years or so, there have been considerable advances in applications of the direct Liapunov method to hydrodynamical stability problems, along two different lines. The first consists of sufficient conditions for stability, following the work of Fjørtoft (1950) and Arnol'd (1965, 1966). The second consists of sufficient conditions for instability, generalizing the classical results of analytical mechanics based on a virial functional (Chetaev 1955; Moiseev and Rumyantsev 1965).

The Fjørtoft-Amol'd stability method has been successfully applied to a number of problems of geophysical interest, including barotropic and baroclinic

Corresponding author address: Dr. T. Shepherd, Department of Physics, University of Toronto, Toronto, MSS 1A7 Canada. quasigeostrophic flow (e.g., Holm et al. 1985; McIntyre and Shepherd 1987; Shepherd 1988a,b, 1989), stratified equilibria (Vladimirov 1985, 1986a), shallow-water flow (Ripa 1983), and axisymmetric homogeneous flow (Vladimirov 1986b; Szeri and Holmes 1988; Shepherd 1991). Results based on the application of the direct Liapunov method to the problem of instability have been, by contrast, rather limited (Ooyama 1966; Vladimirov 1989, 1990; Vladimirov and Rumyantser 1990).

A classical problem in atmospheric dynamics is that of symmetric stability. While the original interest in the problem arose in the context of axisymmetric planetary circulations (e.g., see Charney 1973), there has been considerable recent interest in the possibility that mesoscale rainbands may be a manifestation of (moist) symmetric instability (e.g., Bennetts and Hoskins 1979). Applications of the direct Liapunov method to the problem of symmetric stability go back to Fjørtoft (1950), who showed by a variational method that a sufficient condition for symmetric stability of a basic flow is static stability together with positivity of $f$ times the potential vorticity, and to Ooyama (1966), who employed a virial functional to obtain a one-sided estimate of the growth rate of unstable disturbances.

In this paper, we reexamine the problem of symmetric stability and obtain two main results. The first is an extension of the Fjørtoft (1950) stability theorem to finite-amplitude disturbances, including explicit bounds on the possible growth of unstable disturbances. The second is a two-sided (upper and lower) estimate 
of the growth rate of symmetric instabilities, improving on the results of Ooyama (1966).

The plan of the paper is as follows. In section 2 , the governing equations are described, and their Hamiltonian structure established. Then the stability of a baroclinic basic flow is considered, and Fjørtoft's theorem is recovered within the context of an energy-Casimir (or pseudoenergy) stability analysis. These results are extended to moist adiabatic (saturated) systems. The finite-amplitude version of the stability theorem is derived in section 3 . In section 4 , this finite-amplitude stability theorem is used to obtain rigorous saturation bounds on the growth of disturbances to symmetrically unstable basic flows following the method of Shepherd (1988a). These bounds are compared with parcel-based estimates. The question of symmetric instability is also the subject of section 5 , which derives sufficient conditions for instability and establishes two-sided estimates of the growth rate. Finally in section 6, we briefly consider the problem of the instability of a moist atmosphere with saturated ascent and unsaturated descent. The paper concludes with a discussion.

\section{Classical symmetric stability}

\section{a. Governing equations}

We consider the nonhydrostatic, adiabatic, Boussinesq equations on an $f$ plane:

$$
\begin{gathered}
\frac{D u}{D t}-f v=-\frac{p_{x}}{\rho_{0}}, \\
\frac{D v}{D t}+f u=-\frac{p_{y}}{\rho_{0}}, \\
\frac{D w}{D t}=-\frac{p_{z}}{\rho_{0}}+\frac{g \theta}{\theta_{0}}, \\
u_{x}+v_{y}+w_{z}=0, \\
\frac{D \theta}{D t}=0 .
\end{gathered}
$$

The notation is standard: $p$ is the pressure, $\rho_{0}$ is a constant reference density, $\theta$ is the potential temperature, $\theta_{0}$ is a constant reference potential temperature, $f$ is the constant Coriolis parameter, subscripts denote partial derivatives, and $D / D t \equiv \partial / \partial t+\mathrm{v} \cdot \nabla$. We consider the system under so-called "symmetric" conditions where all dynamical fields are independent of $y$, so that all $y$ derivatives drop out. Equations (2.1a) $-(2.1 \mathrm{e})$ can then be cast in the form

$$
\begin{gathered}
\frac{D u}{D t}=-p_{x}^{*}+f m, \\
\frac{D m}{D t}=0,
\end{gathered}
$$

$$
\begin{gathered}
\frac{D w}{D t}=-p_{z}^{*}+\frac{g \theta}{\theta_{0}}, \\
u_{x}+w_{z}=0, \\
\frac{D \theta}{D t}=0,
\end{gathered}
$$

where $m \equiv v+f x$ is the $y$ component of absolute velocity and $p^{*} \equiv\left(p / \rho_{0}\right)+\frac{1}{2} f^{2} x^{2}$ is a modified pressure. The symmetry in $y$ thus means that the system gains a new Lagrangian invariant $m$, in addition to $\theta$, and $m$ exerts a centrifugal body force in $x$ just as $\theta$ exerts a gravitational body force in $z$.

In light of the nondivergent nature of the velocity field in the $x-z$ plane, it is convenient to introduce a streamfunction $\psi$ defined by

$$
u=\psi_{z}, \quad w=-\psi_{x}
$$

it follows that $D / D t=\partial / \partial t+\partial(\psi, \cdot)$, where $\partial(f, g)$ $\equiv f_{z} g_{x}-f_{x} g_{z}$ is the two-dimensional Jacobian operator. Also, the modified pressure may be eliminated by taking the curl of $(2.2 \mathrm{a}, \mathrm{c})$, which yields a prognostic equation for the vorticity $\omega \equiv u_{z}-w_{x}$. With these definitions, the complete set of nonlinear governing equations reduces to

$$
\begin{gathered}
\omega_{t}=-\partial(\psi, \omega)+\partial(m, f x)+\partial\left(\theta, \frac{g z}{\theta_{0}}\right), \\
m_{t}=-\partial(\psi, m), \quad \theta_{t}=-\partial(\psi, \theta) .
\end{gathered}
$$

Equations (2.4a) $-(2.4 \mathrm{c})$ are considered in a simply connected domain $D$ with the nonpenetrative boundary condition

$$
\psi=0 \quad \text { on } \quad \partial D
$$

if the boundary $\partial D$ is at infinity then $(2.4 \mathrm{~d})$ holds in an appropriate limiting sense.

\section{b. Hamiltonian structure}

The system (2.4) is readily seen to be Hamiltonian, as follows. First note that under the assumed boundary condition, the dynamics described by (2.4) conserves an "energy" integral

$$
\mathscr{H}=\iint_{D}\left\{\frac{1}{2}|\nabla \psi|^{2}-m f x-\frac{\theta g z}{\theta_{0}}\right\} d x d z .
$$

To establish the Hamiltonian structure of the dynamics, it is necessary to evaluate the functional derivatives of $\mathscr{H}$ with respect to $\omega, m$, and $\theta$. To this end,

$$
\begin{aligned}
\delta \mathscr{H} & =\iint_{D}\left\{\nabla \psi \cdot \nabla \delta \psi-f x \delta m-\frac{g z}{\theta_{0}} \delta \theta\right\} d x d z \\
& =\iint_{D}\left\{-\psi \delta \omega-f x \delta m-\frac{g z}{\theta_{0}} \delta \theta\right\} d x d z,
\end{aligned}
$$

the second line following upon integration by parts 
under the presumed boundary condition on $\psi$. It follows immediately from (2.6) that

$$
\frac{\delta \mathscr{H}}{\delta \omega}=-\psi, \quad \frac{\delta \mathscr{H}}{\delta m}=-f x, \quad \frac{\delta \mathscr{H}}{\delta \theta}=-\frac{g z}{\theta_{0}} .
$$

It is now easy to verify that the dynamics (2.4) is equivalently represented in the symplectic (Hamiltonian) form

$$
\mathbf{x}_{t}=J \frac{\delta \mathscr{H}}{\delta \mathbf{x}},
$$

with the identification

$$
J \equiv\left(\begin{array}{ccc}
-\partial(\omega, \cdot) & -\partial(m, \cdot) & -\partial(\theta, \cdot) \\
-\partial(m, \cdot) & 0 & 0 \\
-\partial(\theta, \cdot) & 0 & 0
\end{array}\right) .
$$

For further discussion of the symplectic representation (2.8) in the fluid-dynamical context, one may refer to Benjamin (1984), Salmon (1988), or Shepherd (1990). Of course, merely casting the governing equations into the form (2.8) is not enough to establish Hamiltonian structure; one must also verify certain algebraic properties about the Poisson bracket induced by $J$. In this case, however, the system is a straightforward generalization of 2D Boussinesq stratified flow (obtained by removing $m$ from the above representation), which has been extensively discussed by Benjamin (1986). Hence, such detailed verification is unnecessary.

In addition to the Hamiltonian (2.5), the system (2.4) conserves a family of invariants of the form

$$
\mathcal{e}=\iint_{D} C(m, \theta) d x d z
$$

for arbitrary functions $C(\eta, \xi)$. These are what are known as Casimir invariants, since it is easily verified that

$$
J \frac{\delta \mathcal{C}}{\delta \mathbf{x}}=0
$$

after using

$$
\frac{\delta \mathcal{C}}{\delta \omega}=0, \quad \frac{\delta \mathcal{C}}{\delta m}=C_{m}, \quad \frac{\delta \mathcal{C}}{\delta \theta}=C_{\theta}
$$

(subscripts denoting partial derivatives, as always). The fact that $\mathcal{C}$ is an invariant of the motion is also immediately apparent from the fact that $m$ and $\theta$ are Lagrangian (or material) invariants.

It may seem odd that the functional $\mathscr{H}$ used as the Hamiltonian does not contain the usual $y$ component of kinetic energy, namely, $\frac{1}{2} v^{2}$ but rather $-m f x$; however, the two quantities differ by $\frac{1}{2} m^{2}+\frac{1}{2} f^{2} x^{2}$, the first of which (when integrated over $D$ ) is a Casimir, and the second of which is a constant (also a Casimir, of a trivial sort). Thus, the invariance of $\mathscr{H}$ is equivalent to the invariance of the usual energy. [In any case it is evident from (2.8) and (2.11) that the Hamiltonian is only defined to within a Casimir.] This issue is discussed further in section 4 .

\section{c. Disturbances to a baroclinic basic flow}

We consider the problem of the stability to symmetric disturbances of a baroclinic basic flow

$$
v=V(x, z), \quad \theta=\Theta(x, z), \quad u=0=w,
$$

in thermal wind balance:

$$
f \frac{\partial V}{\partial z}=\frac{g}{\theta_{0}} \frac{\partial \Theta}{\partial x} .
$$

The problem is approached in a very general manner by using the Fjørtoft-Arnol'd stability method based on an energy-Casimir invariant constructed from (2.5) and (2.10) (e.g., see Holm et al. 1985; Shepherd 1990, section 6). Any stability result will therefore apply not only to normal-mode disturbances but also to the continuous spectrum. It will also turn out that the result is immediately generalizable to finite amplitude.

The goal is to establish the convexity of the functional $\mathscr{H}+\mathcal{C}$ in the vicinity of the steady basic flow $(V, \Theta)$. To this end, we first choose the arbitrary function $C$ in (2.10) so that the basic flow is a conditional extremum of $\mathscr{H}+\mathcal{C}$, namely, so that

$$
\frac{\delta \mathscr{H}}{\delta \mathbf{x}}=-\frac{\delta \mathcal{C}}{\delta \mathbf{x}}
$$

when evaluated at the basic state (2.13). [This is always possible since the basic state is presumed to be steady; e.g., see Shepherd (1990, section 5).] Using (2.7) and (2.12), it follows that the appropriate choice of $C$ must satisfy

$$
C_{m}(M, \Theta)=f x, \quad C_{\theta}(M, \Theta)=\frac{g z}{\theta_{0}},
$$

where $M \equiv V+f x$. To determine whether the basic flow is a true extremum, we examine the second variation

$$
\begin{aligned}
\delta^{2}(\mathscr{H}+\mathcal{C})= & \iint_{D}\left\{|\nabla \delta \psi|^{2}+C_{m m}(\delta m)^{2}\right. \\
& \left.+2 C_{m \theta} \delta m \delta \theta+C_{\theta \theta}(\delta \theta)^{2}\right\} d x d z .
\end{aligned}
$$

Now, the kinetic energy part of (2.17) is evidently positive definite, while the remaining terms are also positive definite if

$$
C_{m m}>0, \quad C_{\theta \theta}>0
$$

and

$$
C_{m m} C_{\theta \theta}-\left(C_{m \theta}\right)^{2}>0
$$

when evaluated at the basic flow. Using the relations (2.16) and noting that $x$ and $z$ can in general be re- 
garded as functions of $M$ and $\Theta$, these conditions take the form

$$
f \frac{\partial x}{\partial M}>0, \quad \frac{g}{\theta_{0}} \frac{\partial z}{\partial \Theta}>0
$$

and

$f \frac{\partial x}{\partial M} \frac{g}{\theta_{0}} \frac{\partial z}{\partial \Theta}-f \frac{\partial x}{\partial \Theta} \frac{g}{\theta_{0}} \frac{\partial z}{\partial M}=\frac{f g}{\theta_{0}}\left|\frac{\partial(x, z)}{\partial(M, \Theta)}\right|>0$.

[Condition (2.21) also guarantees that the coordinate transformation from $(M, \Theta)$ to $(x, z)$ is nonsingular.] Thus, whenever the basic flow satisfies $(2.20)$ and $(2.21)$, it follows that the functional $\delta^{2}(\mathscr{H}+\mathcal{C})$ is positive definite for arbitrary disturbances $\delta m, \delta \theta$. Insofar as $\delta^{2}(\mathscr{H}+\mathcal{C})$ is an exact invariant of the linearized equations, this establishes stability within the framework of the linearized system.

Conditions (2.20) and (2.21) have a clear physical interpretation: $(2.20 \mathrm{a}, \mathrm{b})$ establish inertial and static stability, respectively, while (2.21) is equivalent to $f$ times the basic-flow potential vorticity $Q$ being everywhere positive; namely,

$$
\begin{array}{r}
f Q \equiv f \mathbf{Z} \cdot \nabla \Theta=f\left(f+\frac{\partial V}{\partial x}\right) \frac{\partial \Theta}{\partial z}-f \frac{\partial V}{\partial z} \frac{\partial \Theta}{\partial x} \\
=f\left|\frac{\partial(M, \Theta)}{\partial(x, z)}\right|>0,
\end{array}
$$

where $\mathbf{Z}$ is the basic-flow absolute vorticity. Introducing the notation $\zeta \equiv f+V_{x}$, the vertical component of $\mathbf{Z}$, and $N^{2} \equiv g \Theta_{z} / \theta_{0}$, the square of the Brunt-Väisälä frequency, one has

$$
Q=\frac{f \theta_{0} N^{2}}{g}\left(\frac{\zeta}{f}-\frac{V_{z}^{2}}{N^{2}}\right),
$$

after using (2.14). Thus, condition (2.22) is equivalently represented as

$$
\mathrm{Ri} \equiv \frac{N^{2}}{V_{z}^{2}}>\frac{f}{\zeta}>0
$$

where $\mathrm{Ri}$ is the Richardson number. In the special case $V=V(z),(2.24)$ reduces to $\mathrm{Ri}>1$.

Hence we have recovered the sufficient conditions for symmetric stability derived by Fjørtoft (1950), Stone (1966), and Hoskins (1974). While the latter two references considered only normal-mode disturbances, Fjørtoft's analysis treated small-amplitude disturbances of arbitrary form, as here. It is apparent that the stability criteria are based at root on the global invariants of the problem.

\section{d. Moist adiabatic systems}

In a moist adiabatic (saturated) process, the total energy of the system is conserved if the latent heat stored in water vapor is considered as a form of potential energy. We therefore expect the above theory to be applicable to such a system. The governing equations are as given by (2.4), except that $(2.4 \mathrm{c})$ is replaced by conservation of the equivalent potential temperature $\theta_{e}$ :

$$
\theta_{e, t}=-\partial\left(\psi, \theta_{e}\right)
$$

Note that $\theta_{e}=\theta_{e}(\theta, z)$.

We take as the Hamiltonian

$$
\mathscr{H}=\iint_{D}\left\{\frac{1}{2}|\nabla \psi|^{2}-m f x-h\left(\theta_{e}, z\right)\right\} d x d z,
$$

with the function $h$ (representing the negative of the gravitational plus latent potential energy) satisfying the relation

$$
\partial\left(\theta_{e}, \frac{\partial h}{\partial \theta_{e}}\right)=\partial\left(\theta, \frac{g z}{\theta_{0}}\right)
$$

equivalently

$$
\frac{\partial^{2} h}{\partial z \partial \theta_{e}}=\frac{g}{\theta_{0}} \frac{\partial \theta / \partial x}{\partial \theta_{e} / \partial x}
$$

It is easy to verify that the governing equations for the moist adiabatic system are recovered from the symplectic form (2.8) with the identification

$$
\begin{gathered}
\mathbf{x} \equiv\left(\omega, m, \theta_{e}\right)^{T} \\
J \equiv\left(\begin{array}{ccc}
-\partial(\omega, \cdot) & -\partial(m, \cdot) & -\partial\left(\theta_{e}, \cdot\right) \\
-\partial(m, \cdot) & 0 & 0 \\
-\partial\left(\theta_{e}, \cdot\right) & 0 & 0
\end{array}\right) .
\end{gathered}
$$

The Casimir invariants are likewise of the form

$$
\mathcal{e}=\iint_{D} C\left(m, \theta_{e}\right) d x d z
$$

for arbitrary functions $C(\eta, \xi)$.

Following the stability analysis of the previous subsection, this case yields

$$
C_{m}\left(M, \Theta_{e}\right)=f x, \quad C_{\theta_{e}}\left(M, \Theta_{e}\right)=\frac{\partial h}{\partial \Theta_{e}},
$$

where $\Theta_{e}$ is the equivalent potential temperature of the basic state, and the partial derivative of $h$ is taken at constant $z$. Stability of the basic state may be seen to follow provided

$$
C_{m m}=f \frac{\partial x}{\partial M}>0, \quad\left(C_{\theta_{e} \theta_{e}}-\frac{\partial^{2} h}{\partial \Theta_{e}^{2}}\right)=\frac{\partial^{2} h}{\partial z \partial \Theta_{e}} \frac{\partial z}{\partial \Theta_{e}}>0
$$


and

$$
\begin{aligned}
C_{m m}\left(C_{\theta_{e} \theta_{e}}-\frac{\partial^{2} h}{\partial \Theta_{e}^{2}}\right) & -\left(C_{m \theta_{e}}\right)^{2} \\
= & f \frac{\partial^{2} h}{\partial z \partial \Theta_{e}}\left|\frac{\partial(x, z)}{\partial\left(M, \Theta_{e}\right)}\right|>0 .
\end{aligned}
$$

Now, from (2.28) together with the fact that $\theta_{e}=\theta_{e}(\theta$, $z$ ) and $\partial \Theta_{e} / \partial \Theta>0$, it is clear that $\partial^{2} h / \partial z \partial \Theta_{e}>0$; hence, the stability conditions (2.32b) and (2.33) amount to static stability and positive (moist) equivalent potential vorticity (for $f>0$ ), as shown previously by Bennetts and Hoskins (1979) for the special case of normal-mode disturbances.

\section{Nonlinear symmetric stability}

The nature of the analysis performed above shows that the problem of the stability of a baroclinic jet to symmetric disturbances is in many ways analogous to the problem of the stability of a stably stratified resting state (Vladimirov 1987). This makes a certain amount of physical sense, as symmetric disturbances do not feel the vertical shear in the jet directly but only indirectly via the associated lateral potential-temperature gradient. It also means that the linearized result is likely to lead to a nonlinear stability theorem.

We thus assume that the formal stability criteria (2.20) and (2.21) are satisfied and seek an explicit demonstration of nonlinear stability. The problem in this context is to find an exact disturbance invariant that can be sandwiched between disturbance norms. The obvious candidate is $\mathscr{H}+\mathcal{C}$ itself, less its value at the basic state, namely

$\mathcal{A} \equiv(\mathscr{H}+\mathcal{C})[\omega, m, \theta]-(\mathscr{H}+\mathcal{C})[0, M, \Theta]$.

By choosing $\mathcal{C}$ according to (2.15) it follows that $\mathcal{A}$ is of at least quadratic order in disturbance amplitude in the small-amplitude limit. It is also an exact finite-amplitude invariant of the dynamics, since each term in (3.1) is itself an invariant.

Writing the disturbed flow as

$$
\omega=\omega^{\prime}, \quad m=M+m^{\prime}, \quad \theta=\Theta+\theta^{\prime},
$$

with the primed variables not assumed to be of small amplitude, and using (2.16), $\mathcal{A}$ takes the form

$$
\begin{array}{r}
\mathcal{A}=\iint_{D}\left\{\frac{1}{2}\left|\nabla \psi^{\prime}\right|^{2}+C\left(M+m^{\prime}, \Theta+\theta^{\prime}\right)\right. \\
\left.-C(M, \Theta)-C_{m}(M, \Theta) m^{\prime}-C_{\theta}(M, \Theta) \theta^{\prime}\right\} \\
\times d x d z .
\end{array}
$$

Taylor's remainder theorem implies that at each point in space there exists some $\tilde{m} \in\left(M, M+m^{\prime}\right)$ and $\tilde{\theta}$ $E\left(\Theta, \Theta+\theta^{\prime}\right)$ such that

$$
\begin{aligned}
& I \equiv C\left(M+m^{\prime}, \Theta+\theta^{\prime}\right)-C(M, \Theta)-C_{m}(M, \Theta) m^{\prime} \\
& \quad-C_{\theta}(M, \Theta) \theta^{\prime}=\frac{1}{2}\left\{C_{m m}(\tilde{m}, \tilde{\theta}) m^{\prime 2}\right. \\
& \left.\quad+2 C_{m \theta}(\tilde{m}, \tilde{\theta}) m^{\prime} \theta^{\prime}+C_{\theta \theta}(\tilde{m}, \tilde{\theta}) \theta^{\prime 2}\right\}
\end{aligned}
$$

It is straightforward to verify that, in light of (2.18), the quadratic form (3.4) may be bounded from above and below according to

$$
\begin{aligned}
2 I \leqslant\left(\tilde{C}_{m m}+\frac{\theta_{0}}{m_{0}}\left|\tilde{C}_{m \theta}\right|\right) m^{\prime 2} & \\
& +\left(\tilde{C}_{\theta \theta}+\frac{m_{0}}{\theta_{0}}\left|\tilde{C}_{m \theta}\right|\right) \theta^{\prime 2} \\
2 I \geqslant\left(\tilde{C}_{m m}-\frac{\theta_{0}}{m_{0}}\left|\tilde{C}_{m \theta}\right|\right) m^{\prime 2} & \\
& +\left(\tilde{C}_{\theta \theta}-\frac{m_{0}}{\theta_{0}}\left|\tilde{C}_{m \theta}\right|\right) \theta^{\prime 2}
\end{aligned}
$$

where the tilde indicates that the function is evaluated at $(\tilde{m}, \tilde{\theta})$, and $m_{0}$ is some arbitrary positive parameter with the dimensions of $m$, to be chosen later.

Now, using the usual rules for transforming partial derivatives, together with the relations $(2.16)$ and (2.22), we have

$$
\begin{aligned}
C_{m m} & =f \frac{\partial x}{\partial M}=\frac{f}{Q} \frac{\partial \Theta}{\partial z}, \\
C_{\theta \theta} & =\frac{g}{\theta_{0}} \frac{\partial z}{\partial \Theta}=\frac{g}{\theta_{0} Q} \frac{\partial M}{\partial x}, \\
C_{m \theta} & =f \frac{\partial x}{\partial \Theta}=-\frac{f}{Q} \frac{\partial M}{\partial z}, \\
& =\frac{g}{\theta_{0}} \frac{\partial z}{\partial M}=-\frac{g}{\theta_{0} Q} \frac{\partial \Theta}{\partial x} .
\end{aligned}
$$

Without serious loss of generality we assume $f \Theta_{x}>0$, corresponding to $V_{z}>0$; since $f Q>0$ by hypothesis, this implies that $C_{m \theta}<0$. Substituting (3.6) into (3.5) then yields

$$
\begin{array}{r}
2 I \leqslant\left(\frac{f}{Q} \frac{\partial \Theta}{\partial z}+\frac{f \theta_{0}}{m_{0} Q} \frac{\partial M}{\partial z}\right) m^{\prime 2} \\
+\left(\frac{g}{\theta_{0} Q} \frac{\partial M}{\partial x}+\frac{m_{0} g}{\theta_{0}^{2} Q} \frac{\partial \Theta}{\partial x}\right) \theta^{\prime 2} \\
2 I \geqslant\left(\frac{f}{Q} \frac{\partial \Theta}{\partial z}-\frac{f \theta_{0}}{m_{0} Q} \frac{\partial M}{\partial z}\right) m^{\prime 2} \\
+\left(\frac{g}{\theta_{0} Q} \frac{\partial M}{\partial x}-\frac{m_{0} g}{\theta_{0}^{2} Q} \frac{\partial \Theta}{\partial x}\right) \theta^{\prime 2}
\end{array}
$$


it being understood that the various basic-flow functions are evaluated at some $(\tilde{m}, \tilde{\theta})$. If one now makes the choice $m_{0}=g / N$ (possibly a function of $x$ and $z$ ), then (3.7) reduce to the compact forms

$$
\begin{aligned}
& 2 I \leqslant \frac{f \theta_{0} N^{2}}{g Q}\left\{\left(1+\frac{V_{z}}{N}\right) m^{\prime 2}+\left(\frac{\zeta}{f}+\frac{V_{z}}{N}\right) \frac{g^{2}}{N^{2} \theta_{0}^{2}} \theta^{\prime 2}\right\}, \\
& 2 I \geqslant \frac{f \theta_{0} N^{2}}{g Q}\left\{\left(1-\frac{V_{z}}{N}\right) m^{\prime 2}+\left(\frac{\zeta}{f}-\frac{V_{z}}{N}\right) \frac{g^{2}}{N^{2} \theta_{0}^{2}} \theta^{\prime 2}\right\} .
\end{aligned}
$$

We now use the inequalities (3.8a) and (3.8b) to obtain a priori estimates (upper and lower bounds) on $\mathcal{A}$. Given some basic flow satisfying (2.20) and (2.21), suppose constants $c^{-}, c^{+}$exist such that

$$
\begin{gathered}
\frac{f \theta_{0} N^{2}}{g Q} \min \left\{\left(1-\frac{V_{z}}{N}\right),\left(\frac{\zeta}{f}-\frac{V_{z}}{N}\right)\right\} \geqslant c^{-}>0, \\
\frac{f \theta_{0} N^{2}}{g Q} \max \left\{\left(1+\frac{V_{z}}{N}\right),\left(\frac{\zeta}{f}+\frac{V_{z}}{N}\right)\right\} \leqslant c^{+}<\infty .
\end{gathered}
$$

[This is actually somewhat more restrictive than (2.20), (2.21).] Now, the basic-flow quantities in (3.8a) and (3.8b) are evaluated at some unknown values of $\tilde{m}$ $\in\left(M, M+m^{\prime}\right)$ and $\tilde{\theta} \in\left(\Theta, \Theta+\theta^{\prime}\right)$. If the disturbance is "natural" in the sense that no new values of $m$ and $\theta$ are introduced (recall that they are Lagrangian invariants), then the inequalities (3.9) are sufficient to bound the expressions in (3.8a) and (3.8b). If the disturbance does introduce values of $m$ and $\theta$ outside the range of $M$ and $\Theta$, then the basic-flow functional relations may be arbitrarily extended to cover those new values in such a way as to satisfy (3.9) (cf. Arnol'd 1966). Either way, the estimates

$$
\begin{aligned}
& \mathcal{A} \leqslant \iint_{D}\left\{\frac{1}{2}\left|\nabla \psi^{\prime}\right|^{2}+\frac{c^{+}}{2}\left(m^{\prime 2}+\frac{g^{2}}{N^{2} \theta_{0}^{2}} \theta^{\prime 2}\right)\right\} d x d z \\
& \mathcal{A} \geqslant \iint_{D}\left\{\frac{1}{2}\left|\nabla \psi^{\prime}\right|^{2}+\frac{c^{-}}{2}\left(m^{\prime 2}+\frac{g^{2}}{N^{2} \theta_{0}^{2}} \theta^{\prime 2}\right)\right\} d x d z
\end{aligned}
$$

are obtained. This essentially establishes normed stability. To demonstrate this fact explicitly, define the disturbance norm $\left\|\mathbf{x}^{\prime}\right\|_{\lambda}$ by

$$
\begin{array}{r}
\left\|\mathbf{x}^{\prime}\right\|_{\lambda}^{2} \equiv \iint_{D}\left\{\frac{1}{2}\left|\nabla \psi^{\prime}\right|^{2}+\frac{\lambda}{2}\left(m^{\prime 2}+\frac{g^{2}}{N^{2} \theta_{0}^{2}} \theta^{\prime 2}\right)\right\} \\
\times d x d z,
\end{array}
$$

with $\lambda \in\left[c^{-}, c^{+}\right]$. Then $\left\|\mathbf{x}^{\prime}\right\|_{\lambda}$ at any time $t$ is bounded in terms of its initial value according to

$$
\begin{aligned}
& \left\|\mathbf{x}^{\prime}(t)\right\|_{\lambda}^{2} \\
& \leqslant \frac{\lambda}{c^{-}} \iint_{D}\left\{\frac{1}{2}\left|\nabla \psi^{\prime}\right|^{2}+\frac{c^{-}}{2}\left(m^{\prime 2}+\frac{g^{2}}{N^{2} \theta_{0}^{2}} \theta^{\prime 2}\right)\right\}(t) \\
& \times d x d z \leqslant \frac{\lambda}{c^{-}} \mathcal{A}(t)=\frac{\lambda}{c^{-}} \mathcal{A}(0) \\
& \leqslant \frac{\lambda}{c^{-}} \iint_{D}\left\{\frac{1}{2}\left|\nabla \psi^{\prime}\right|^{2}+\frac{c^{+}}{2}\left(m^{\prime 2}+\frac{g^{2}}{N^{2} \theta_{0}^{2}} \theta^{\prime 2}\right)\right\}(0) \\
& \times d x d z \leqslant \frac{c^{+}}{c^{-}}\left\|\mathbf{x}^{\prime}(0)\right\|_{\lambda}^{2} .
\end{aligned}
$$

The chain of inequalities (3.12) establishes that the disturbance "amplification factor," as measured in the norm defined by $(3.11)$, is bounded by $\left(c^{+} / c^{-}\right)^{1 / 2}$, with $c^{+}$and $c^{-}$chosen so as to satisfy (3.9). Note that if $Q \rightarrow 0$ somewhere in the basic flow, the factor $\left(c^{+} /\right.$ $\left.c^{-}\right)^{1 / 2}$ diverges and normed stability is lost.

In the special case where $V=V(z), \zeta=f$, and we may take

$$
\frac{c^{+}}{c^{-}}=\frac{\left(1+\frac{V_{z}}{N}\right)_{\max }}{\left(1-\frac{V_{z}}{N}\right)_{\min }}=\frac{\mathrm{Ri}_{\min }^{1 / 2}+1}{\mathrm{Ri}_{\text {min }}^{1 / 2}-1} .
$$

As expected, the maximum amplification is seen to diverge as $R i_{\text {min }} \rightarrow 1$.

It is clear from the nature of the above analysis that the stability theorem for moist adiabatic systems may also be extended to finite amplitude, although the details concerning the amplification factor will be somewhat more complicated.

\section{Nonlinear saturation of symmetric instability \\ a. General theory}

In this section we consider the following problem. Given an infinitesimal disturbance to some unstable steady flow, can a nontrivial upper bound be obtained on the saturation amplitude of the instability? This question is addressed following the method of Shepherd (1988a), using the nonlinear stability theorem (3.12).

Thus, the initial condition of the system (2.4) takes the form

$$
\begin{array}{r}
m(0)=m^{(0)}+m^{(1)}, \quad \theta(0)=\theta^{(0)}+\theta^{(1)}, \\
\omega(0)=\omega^{(1)},
\end{array}
$$

where $\left(m^{(0)}, \theta^{(0)}\right)$ is some steady state that is known to be unstable, and the initial perturbation $\left(m^{(1)}, \theta^{(1)}\right.$, $\left.\omega^{(1)}\right)$ is of infinitesimal amplitude. (The restriction to initially infinitesimal disturbances is not necessary, but it represents an important subclass of such problems.) We now consider the initial condition (4.1) as a (finiteamplitude) disturbance to some stable basic flow ( $M$, 
$\Theta)$, as in (3.2). The basic flow is completely arbitrary apart from the fact that it is presumed to satisfy (3.9) for some constants $c^{+}, c^{-}$(which are then functionals of the choice of the basic flow). For any such choice of a stable basic flow, the inequality (3.12) then provides an upper bound on the disturbance norm (3.11) in terms of its initial value.

What we seek is an upper bound on the perturbation amplitude, and the disturbance norm (3.11) is not a suitable measure of this since it depends on the choice of the stable basic flow $(M, \Theta)$. An unambiguous estimate of perturbation amplitude, however, is provided by the kinetic energy of the motion in the $x-z$ plane, and in this respect we have the obvious inequality

$$
\begin{aligned}
& \iint_{D} \frac{1}{2}|\nabla \psi|^{2}(t) d x d z \\
& \quad=\iint_{D} \frac{1}{2}\left|\nabla \psi^{\prime}\right|^{2}(t) d x d z \leqslant\left\|\mathbf{x}^{\prime}(t)\right\|_{\lambda}^{2} .
\end{aligned}
$$

[Recall that the primed quantities are departures from the basic flow $(M, \Theta)$, as in (3.2), and $\psi=0$.] Combining (4.2) with (3.12) yields (upon taking $\lambda=c^{-}$)

$$
\begin{aligned}
& \iint_{D} \frac{1}{2}|\nabla \psi|^{2}(t) d x d z \leqslant \iint_{D}\left\{\frac{1}{2}\left|\nabla \psi^{\prime}\right|^{2}(0)\right. \\
& \left.+\frac{c^{+}}{2}\left(m^{\prime 2}(0)+\frac{g^{2}}{N^{2} \theta_{0}^{2}} \theta^{\prime 2}(0)\right)\right\} d x d z \\
& =\iint_{D} \frac{c^{+}}{2}\left\{\left(m^{(0)}-M\right)^{2}+\frac{g^{2}}{N^{2} \theta_{0}^{2}}\left(\theta^{(0)}-\Theta\right)^{2}\right\} d x d z \\
& +\mathcal{O}(a),
\end{aligned}
$$

where $a \ll 1$ is some measure of the initial perturbation amplitude; to get to the final line of (4.3), the fact that

$$
\begin{array}{r}
\psi^{\prime}(0)=\mathcal{O}(a), \quad m^{\prime}(0)=m^{(0)}-M+\mathcal{O}(a), \\
\theta^{\prime}(0)=\theta^{(0)}-\Theta+\mathcal{O}(a)
\end{array}
$$

has been used. The inequality (4.3) represents a rigorous upper bound on the saturation amplitude of the instability for any choice of a stable basic flow, the right-hand side of which is a functional of the basic flow (including $c^{+}$and $N^{2}$ ). One may therefore consider the optimization problem of minimizing the righthand side of (4.3) in order to obtain the tightest (i.e., most constraining) upper bound.

\section{b. Example: pure baroclinic flow}

To make the above theory concrete, we consider the example of a pure baroclinic flow

$$
\begin{aligned}
& v^{(0)}=\tilde{N}(1+\epsilon) z, \\
& \theta^{(0)}=\frac{\theta_{0} \tilde{N}^{2}}{g} z+\frac{\theta_{0} \tilde{N} f}{g}(1+\epsilon) x,
\end{aligned}
$$

where $\tilde{N}$ is the (constant) Brunt-Väisälä frequency of the initial flow (4.5). The problem is considered in the rectangular domain

$$
-\frac{L}{2} \leqslant x \leqslant \frac{L}{2}, \quad-\frac{H}{2} \leqslant z \leqslant \frac{H}{2} .
$$

For the flow (4.5), $\mathrm{Ri}=1 /(1+\epsilon)^{2}$, so we expect instability when the "supercriticality" parameter $\epsilon$ is positive.

Now introduce a basic flow defined by

$$
\begin{aligned}
& V=\tilde{N}(1-\delta) z, \\
& \Theta=\frac{\theta_{0} \tilde{N}^{2}}{g}(1+\gamma) z+\frac{\theta_{0} \tilde{N} f}{g}(1-\delta) x,
\end{aligned}
$$

with static stability given by

$$
N^{2} \equiv(1+\gamma) \tilde{N}^{2} .
$$

The basic flow (4.7) is stable to symmetric disturbances provided

$$
\mathrm{Ri}=\frac{(1+\gamma)}{(1-\delta)^{2}}>1 \Leftrightarrow(1-\delta)^{2}<(1+\gamma) .
$$

Using (4.4), (4.5), and (4.7), the initial disturbance fields are given (in the limit $a \rightarrow 0$ ) by

$$
\begin{aligned}
m^{\prime}(0) & =\tilde{N}(\epsilon+\delta) z, \\
\theta^{\prime}(0) & =\frac{\theta_{0} \tilde{N} f}{g}(\epsilon+\delta) x-\frac{\theta_{0} \tilde{N}^{2}}{g} \gamma z .
\end{aligned}
$$

Then substituting (4.10) into the right-hand side of (4.3) leads to the bound

$$
\begin{aligned}
& \iint_{D} \frac{1}{2}|\nabla \psi|^{2}(t) d x d z \leqslant \frac{1}{24} H L c^{+}\left\{\tilde{N}^{2} H^{2}\right. \\
& \left.\quad \times\left[(\epsilon+\delta)^{2}+\frac{\gamma^{2}}{1+\gamma}\right]+f^{2} L^{2} \frac{(\epsilon+\delta)^{2}}{1+\gamma}\right\} .
\end{aligned}
$$

In order to determine $c^{+}$in terms of the basic-flow parameters, note from (2.23) that the basic-flow potential vorticity takes the form

$$
Q=\frac{f \theta_{0} N^{2}}{g}\left(1-\frac{(1-\delta)^{2}}{(1+\gamma)}\right)
$$

whence using (3.9), one may take

$$
c^{+}=\frac{(1+\gamma)+(1+\gamma)^{1 / 2}(1-\delta)}{\gamma+\delta(2-\delta)} .
$$

The goal now is to minimize the right-hand side of the expression (4.11), using (4.13), over all values of $\delta$ and $\gamma$ satisfying (4.9).

Consider first the bound that is obtained by adjusting only the vertical wind shear and not the static stability; that is, with $\gamma=0$ and $\delta$ kept free. In that case $c^{+}$ $=1 / \delta$, and the right-hand side of (4.11) takes the form

$$
\frac{1}{24} H L \frac{(\epsilon+\delta)^{2}}{\delta}\left(\tilde{N}^{2} H^{2}+f^{2} L^{2}\right) \text {. }
$$


The expression (4.14) is minimized for $\delta=\epsilon$, taking the value

$$
\frac{1}{6} H L\left(\tilde{N}^{2} H^{2}+f^{2} L^{2}\right) \epsilon .
$$

The rigorous upper bound represented by $(4.15)$ implies that the saturation amplitude of a symmetric instability is bounded asymptotically by the square root of the supercriticality $\epsilon$ as $\epsilon \rightarrow 0$. This parallels the case of barotropic instability (Shepherd 1988a, section 5). It is a valid bound provided (4.9) holds, which is true so long as $\epsilon<2$.

Consider next the bound that is obtained by adjusting only the static stability and not the wind shear, that is, with $\delta=-\epsilon$ and $\gamma$ kept free. In that case,

$$
c^{+}=\frac{(1+\gamma)+(1+\gamma)^{1 / 2}(1+\epsilon)}{\gamma-\epsilon(2+\epsilon)},
$$

and the right-hand side of (4.11) takes the form

$$
\frac{1}{24} H L \frac{(1+\gamma)+(1+\gamma)^{1 / 2}(1+\epsilon)}{\gamma-\epsilon(2+\epsilon)} \tilde{N}^{2} H^{2} \frac{\gamma^{2}}{1+\gamma} \text {. }
$$

Ideally one should try to minimize (4.17) over all values of $\gamma$ consistent with (4.9), that is, for all

$$
\gamma>\epsilon(2+\epsilon) \text {. }
$$

The expression (4.17) is a little awkward to manipulate directly, however, because of the factor $(1+\gamma)^{1 / 2}$. But since $\gamma>0$ for all $\gamma$ satisfying (4.18) -recall that $\epsilon$ $>0$ by hypothesis-it follows that $(1+\gamma)^{1 / 2}<(1$ $+\gamma)$, and hence the upper bound (4.17) is itself bounded by

$$
\begin{aligned}
& \frac{1}{24} H L \frac{(1+\gamma)+(1+\gamma)(1+\epsilon)}{\gamma-\epsilon(2+\epsilon)} \tilde{N}^{2} H^{2} \frac{\gamma^{2}}{1+\gamma} \\
& =\frac{1}{24} H^{3} L \tilde{N}^{2} \frac{(2+\epsilon) \gamma^{2}}{\gamma-\epsilon(2+\epsilon)} .
\end{aligned}
$$

The expression (4.19) is minimized for $\gamma=2 \epsilon(2+\epsilon)$, the latter condition evidently satisfying (4.18), and takes the value

$$
\frac{1}{6} H^{3} L \tilde{N}^{2} \epsilon(2+\epsilon)^{2} .
$$

As with (4.15), the bound (4.20) demonstrates that for small $\epsilon$, the saturation amplitude of the instability is bounded asymptotically by the square root of $\epsilon$ as $\epsilon \rightarrow 0$.

It is clear that for $f^{2} L^{2} \gg \check{N}^{2} H^{2}$, the bound (4.20) is significantly smaller than the bound (4.15); while for $f^{2} L^{2} \ll \tilde{N}^{2} H^{2},(4.15)$ is smaller than $(4.20)$ by a factor of at least 4 (limiting to 4 as $\epsilon \rightarrow 0$ ). In general, the bound (4.20) is smaller than (4.15) whenever

$$
\frac{f^{2} L^{2}}{\tilde{N}^{2} H^{2}}>3+4 \epsilon+\epsilon^{2} \text {. }
$$

One could presumably find even better (i.e, more constraining) bounds by allowing both $\delta$ and $\gamma$ to vary simultaneously in the minimization calculation. But the algebraic complexity of such a calculation is sufficiently daunting that it does not seem worth the effort in the present context.

It is of interest to compare the bounds (4.15) and (4.20) with general upper bounds such as the total amount of energy in the system. In this regard the Hamiltonian (2.5) is not the appropriate quantity to consider, since it is not positive definite [ and in fact is negative for the initial condition (4.5)]. Instead, we note that

$$
-m f x=\frac{1}{2} v^{2}-\frac{1}{2} m^{2}-\frac{1}{2} f^{2} x^{2}
$$

and

$-\frac{\theta g z}{\theta_{0}}=\frac{1}{2}\left(\frac{\theta g}{\theta_{0} \tilde{N}}-\tilde{N} z\right)^{2}-\frac{1}{2} \frac{g^{2}}{\theta_{0}^{2} \tilde{N}^{2}} \theta^{2}-\frac{1}{2} \tilde{N}^{2} z^{2} ;$

substituting these expressions into (2.5) yields

$$
\begin{array}{r}
\mathscr{H}=\mathscr{E}-\iint_{D} \frac{1}{2}\left\{m^{2}+f^{2} x^{2}+\frac{g^{2}}{\theta_{0}^{2} \tilde{N}^{2}} \theta^{2}+\tilde{N}^{2} z^{2}\right\} \\
\times d x d z
\end{array}
$$

where

$$
\mathscr{E}=\iint_{D} \frac{1}{2}\left\{|\nabla \psi|^{2}+v^{2}+\left(\frac{\theta g}{\theta_{0} \tilde{N}}-\tilde{N} z\right)^{2}\right\} d x d z .
$$

The integral in (4.24) is seen to be a Casimir invariant, which means that $\mathscr{E}$ is also invariant. Since it is also positive definite, it serves as a suitable measure of the energy of the system. Evidently we have the immediate upper bound,

$$
\iint_{D} \frac{1}{2}|\nabla \psi|^{2} d x d z \leqslant \mathscr{E}
$$

and the bounds obtained from (4.11) can only be considered nontrivial if they are less than $\mathscr{E}$.

For the initial condition (4.5), it is straightforward to verify that

$$
\mathscr{E}=\frac{1}{24} H L\left(\tilde{N}^{2} H^{2}+f^{2} L^{2}\right)(1+\epsilon)^{2} .
$$

This is larger than (4.15) for all $\epsilon<1$; therefore, for this range of $\epsilon$ the bound (4.15) provides a nontrivial constraint on the saturation amplitude. On the other hand, (4.27) is larger than (4.20) for all $\epsilon$ such that

$$
\frac{\epsilon(2+\epsilon)^{2}}{(1+\epsilon)^{2}}<\frac{1}{4}\left(1+\frac{f^{2} L^{2}}{\tilde{N}^{2} H^{2}}\right) .
$$


Introducing the parameter

$$
\mu \equiv \frac{f^{2} L^{2}}{\tilde{N}^{2} H^{2}},
$$

the bounds (4.15), (4.20), and (4.27) may be combined together to yield the rigorous bound

$$
\iint_{D} \frac{1}{2}|\nabla \psi|^{2} d x d z \leqslant \frac{1}{24} H^{3} L \tilde{N}^{2} F(\epsilon, \mu)
$$

where

$F(\epsilon, \mu) \equiv\left\{\begin{array}{c}4(1+\mu) \epsilon, 1+\mu \leqslant(2+\epsilon)^{2} \text { and } \epsilon \leqslant 1 \\ 4 \epsilon(2+\epsilon)^{2}, 1+\mu \geqslant(2+\epsilon)^{2} \text { and } \\ 4 \epsilon(2+\epsilon)^{2}(1+\epsilon)^{-2} \leqslant 1+\mu \\ (1+\epsilon)^{2}(1+\mu), \text { otherwise. }\end{array}\right.$

This is a major result of this section.

\section{c. Comparison with heuristic saturation estimates}

The inequality (4.29) obtained above represents a fully rigorous, nonlinear upper bound on the kinetic energy of a symmetric disturbance to the unstable flow (4.5). In particular, there has been no assumption made as to the nature of the equilibrated state-if indeed one even exists. This result may be contrasted with heuristic saturation estimates based on physical presuppositions concerning the behavior of fluid parcels undergoing symmetric instability (Emanuel 1983).

For example, one may estimate the amount of available potential energy released by slantwise convection if one assumes that the parcels act to $\operatorname{mix} \theta$ along $m$ surfaces. This assumption is appropriate in the nonhydrostatic limit $\mu \gg 1$ (i.e., the $\theta$ surfaces slope steeply compared to the aspect ratio of the domain). For the unstable flow (4.5), the initial $\theta$ distribution given by $\theta^{(0)}(x, z)$ is then presumed to evolve to a new distribution

$$
\theta^{(f)}(x, z)=[\tilde{N}(1+\epsilon) z+f x] \frac{\theta_{0} \tilde{N}}{g}(1+\epsilon),
$$

whose isopleths are parallel to the $m^{(0)}$ surfaces implied by (4.5) and whose value on each $m^{(0)}$ surface equals the mean value of $\theta^{(0)}$ along that surface: $\theta^{(f)}=\theta^{(0)}$ for $z=0$ (see Fig. 1). The available potential energy released thereby is given by

$$
\begin{aligned}
& \iint_{D} \frac{g z}{\theta_{0}}\left(\theta^{(f)}-\theta^{(0)}\right) d x d z \\
& =\iint_{D} \tilde{N}^{2}\left[(1+\epsilon)^{2}-1\right] z^{2} d x d z \\
& =\frac{1}{12} H^{3} L \tilde{N}^{2}\left[(1+\epsilon)^{2}-1\right] \\
& \quad=\frac{1}{12} H^{3} L \tilde{N}^{2} \epsilon(2+\epsilon) .
\end{aligned}
$$

This estimate is similar to the rigorous bound (4.20)which is the relevant one for $\mu \gg 1$-but is smaller by a factor of $2(2+\epsilon)$, that is, by a factor of 4 in the limit $\epsilon \rightarrow 0$.

It should be emphasized that the expressions (4.20) and (4.31) are really quite complementary. The expression (4.31) builds in some mathematical assumptions based on physical reasoning in order to ob-
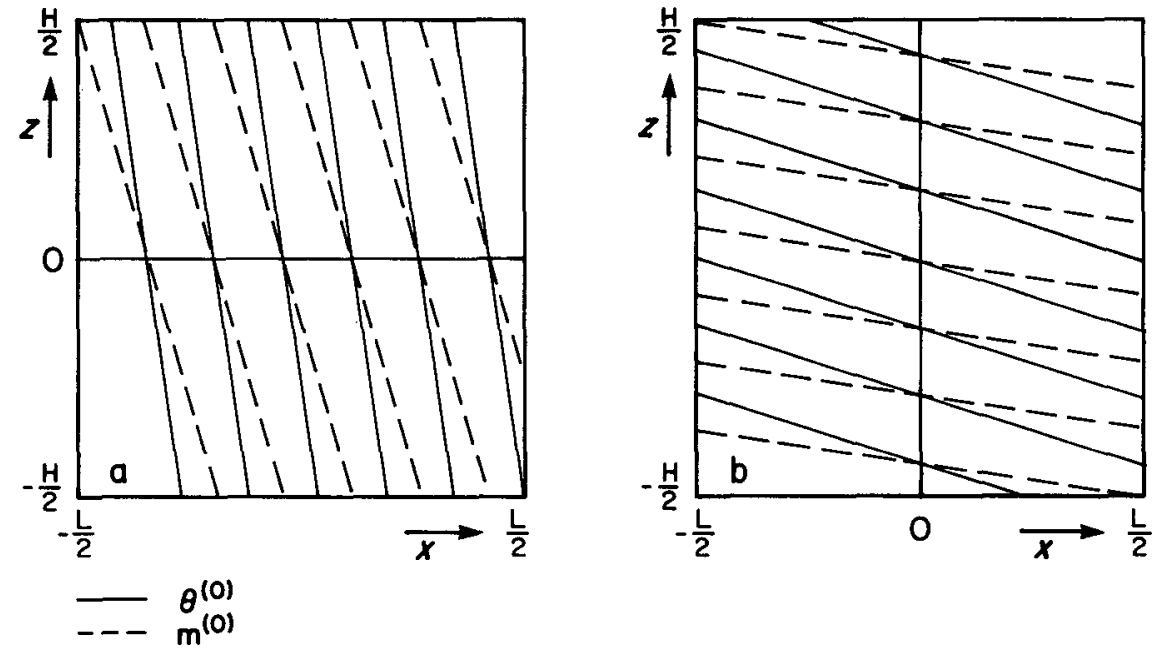

FIG. 1. Sketch of $\theta^{(0)}$ and $m^{(0)}$ isopleths for the unstable basic state (4.5). (a) The nonhydrostatic limit $\mu \gg 1$; in this case mixing of $\theta^{(0)}$ along $m^{(0)}$-surfaces leads to a final distribution $\theta^{(f)}$ such that $\theta^{(\delta)}=\theta^{(0)}$ at $z=0$. (b) The hydrostatic limit $\mu \ll 1$; in this case mixing of $m^{(0)}$ along $\theta^{(0)}$. surfaces leads to a final distribution $m^{(f)}$ such that $m^{(f)}=m^{(0)}$ at $x=0$. 
tain an estimate of the saturation amplitude of the instability; however, there is nothing to prevent the kinetic energy from exceeding that estimate. In contrast, the expression (4.20) is a rigorous upper bound on the saturation amplitude, obtained by manipulation of the global invariants of the fully nonlinear equations; however, there is nothing to ensure that the kinetic energy will come anywhere close to this upper bound. So the upper bound (4.20) provides a rigorous underpinning for the estimate (4.31), while the estimate (4.31) suggests that the upper bound (4.20) should not be a gross overestimate of the actual saturation amplitude. This sort of complementarity is reminiscent of that between saturation bounds and equilibration estimates in barotropic and quasigeostrophic baroclinic instability, which typically also differ by a factor of 4 (Shepherd 1988a, section 7.3; 1989, section 5).

On the other hand, in the hydrostatic limit $\mu \ll 1$ one expects changes in $\theta$ to be negligible, and it is appropriate to estimate the amount of centrifugal potential energy released by slantwise convection by assuming that the parcels act to mix $m$ along $\theta$ surfaces. For the unstable flow (4.5), the initial $m$ distribution given by $m^{(0)}(x, z)$ is presumed to evolve to a new distribution

$$
m^{(f)}(x, z)=[\tilde{N} z+f(1+\epsilon) x](1+\epsilon),
$$

whose isopleths are parallel to the $\theta^{(0)}$ surfaces given by $(4.5)$ and whose value on each $\theta^{(0)}$ surface equals the mean value of $m^{(0)}$ along that surface: $m^{(f)}=m^{(0)}$ for $x=0$ (see Fig. 1). The centrifugal potential energy released thereby is given by

$$
\begin{aligned}
& \iint_{D} f x\left(m^{(f)}-m^{(0)}\right) d x d z \\
& \quad=\iint_{D} f^{2}\left[(1+\epsilon)^{2}-1\right] x^{2} d x d z \\
& =\frac{1}{12} H L^{3} f^{2}\left[(1+\epsilon)^{2}-1\right] \\
& =\frac{1}{12} H L^{3} f^{2} \epsilon(2+\epsilon) .
\end{aligned}
$$

The relevant rigorous upper bound when $\mu \ll 1$ is given by $(4.15)$, which is well approximated in this limit by $\frac{1}{6} H^{3} L \tilde{N}^{2} \epsilon$. The heuristic estimate (4.33) is asymptotically smaller than this by a factor of $\mu$, which suggests that the bound (4.15) may be a considerable overestimate of the saturation amplitude in the hydrostatic limit $\mu \ll 1$.

To obtain parcel-based estimates in the intermediate regime, $\mu=O(1)$ is difficult, due to a lack of knowledge about the slope of the parcel trajectories. In contrast, the upper bound (4.29) is rigorously valid throughout this regime.

\section{A priori estimates of symmetric instability}

\section{a. Linearized equations}

In this section we consider the problem of the instability of the basic flow (2.13) to infinitesimal disturbances. It is convenient to introduce the Lagrange displacement field $(\xi, \eta)$ in the $x-z$ plane defined by (Chandrasekhar 1970)

$$
\xi_{t}=u, \quad \eta_{t}=w, \quad \xi_{x}+\eta_{z}=0 .
$$

Integrating the linearized form of (2.2b) and (2.2e) in time and setting the functions of integration to zero (this amounts to assuming that the disturbance consists only of a rearrangement of the fluid particles) leads to

$$
\theta^{\prime}=-\xi \Theta_{x}-\eta \Theta_{z}, \quad m^{\prime}=-\xi M_{x}-\eta M_{z} .
$$

Using (5.1), (5.2a), and, (5.2b), the linearized form of (2.2a) and (2.2c) may be written as

$$
\begin{aligned}
\xi_{t t} & =-p_{x}^{*}-R_{\xi \xi} \xi-R_{\xi \eta} \eta, \\
\eta_{t t} & =-p_{z}^{*}-R_{\eta \xi} \xi-R_{\eta \eta} \eta,
\end{aligned}
$$

where

$$
\left(\begin{array}{ll}
R_{\xi \xi} & R_{\xi \eta} \\
R_{\eta \xi} & R_{\eta \eta}
\end{array}\right) \equiv\left(\begin{array}{cc}
f M_{x} & f M_{z} \\
\frac{g}{\theta_{0}} \Theta_{x} & \frac{g}{\theta_{0}} \Theta_{z}
\end{array}\right) .
$$

Note that the above tensor is symmetric by virtue of the thermal wind relation (2.14). The equations (5.1) and (5.3) are considered together with the nonpenetrative boundary condition

$$
(\xi, \eta) \cdot \mathbf{n}=0 \quad \text { on } \quad \partial D,
$$

where $\mathbf{n}$ is the unit outward normal. [As with (5.2), the derivation of ( 5.5$)$ from (2.4d) involves setting the functions of integration to zero.] The problem defined above conserves an energy integral, namely,

$$
\dot{\mathscr{E}} \equiv \dot{\mathcal{K}}+\dot{\mathcal{P}}=0
$$

where

$$
\begin{aligned}
& \mathcal{K} \equiv \iint_{D} \frac{1}{2}\left(u^{2}+w^{2}\right) d x d z, \\
& \mathcal{P} \equiv \iint_{D} \frac{1}{2}\left(R_{\xi \xi} \xi^{2}+2 R_{\xi \eta} \xi \eta+R_{\eta \eta} \eta^{2}\right) d x d z,
\end{aligned}
$$

and the dot denotes the time derivative. It may be verified that the energy defined by (5.7a) and (5.7b) is consistent with the expression (2.17) with $\delta m=m^{\prime}$, $\delta \theta=\theta^{\prime}, \nabla \delta \psi=(-w, u)$, after using (5.2a) and (5.2b).

\section{b. Virial functional}

A mean-square measure (norm) of disturbances is introduced according to 


$$
\mathcal{M} \equiv \iint_{D} \frac{1}{2}\left(\xi^{2}+\eta^{2}\right) d x d z,
$$

whose time derivative is the virial functional (Chandrasekhar 1970)

$$
\mathcal{W} \equiv \dot{M}=\iint_{D}(u \xi+w \eta) d x d z
$$

Using the equations of motion together with the fact that $R_{\xi \eta}=R_{\eta \xi}$, it may be shown that

$$
\ddot{M}=\mathscr{\mathcal { W }}=2(\mathcal{K}-\mathscr{P})=4 \mathcal{K}-2 \mathscr{E} ;
$$

this is known as the virial equality (Chandrasekhar 1970).

\section{c. Main inequality}

At this point all the relevant information is contained in the two equalities (5.6) and (5.10). To construct the Liapunov functional, (5.10) is multiplied by an arbitrary constant $\lambda$ and the result subtracted from (5.6); after some manipulation this yields

$$
\dot{\mathscr{E}}_{\lambda}=2 \lambda \mathscr{E}_{\lambda}-4 \lambda \mathcal{K}_{\lambda},
$$

where

$$
\begin{aligned}
\mathscr{E}_{\lambda} \equiv \mathcal{K}_{\lambda}+\mathcal{P}_{\lambda}, \quad \mathcal{P}_{\lambda} \equiv \mathscr{P}+\lambda^{2} \mathcal{M} \\
\mathcal{K}_{\lambda} \equiv \mathscr{K}-\lambda \dot{M}+\lambda^{2} \mathcal{M} \\
=\iint_{D} \frac{1}{2}\left\{(u-\lambda \xi)^{2}+(w-\lambda \eta)^{2}\right\} d x d z .
\end{aligned}
$$

Now suppose that $\lambda>0$. Then, since $\mathscr{K}_{\lambda}$ is evidently non-negative, it follows from (5.11) that

$$
\dot{\mathscr{E}}_{\lambda} \leqslant 2 \lambda \mathscr{E}_{\lambda},
$$

which implies the main inequality

$$
\mathscr{E}_{\lambda}(t) \leqslant \mathscr{E}_{\lambda}(0) e^{2 \lambda t}
$$

It must be emphasized that (5.14) is valid for any solution of the defined problem (5.1), (5.3), and (5.5) and for any positive value of $\lambda$. In particular, no restriction has been placed on the sign of the potential energy functional $\mathscr{P}$. The monotonic change in $\mathscr{E}_{\lambda}$ implied by $(5.14)$ in the case $\mathscr{E}_{\lambda}<0$ suggests that it may be used as a Liapunov functional in the instability problem.

It follows from (5.12) and (5.14) that

$$
\mathscr{P}(t)<\mathscr{E}_{\lambda}(0) e^{2 \lambda t} \text {. }
$$

\section{d. Estimate from below of increase in disturbance amplitude}

Now we suppose that the stability criteria (2.18), (2.19) do not hold; it follows that a displacement field $(\xi, \eta)$ exists for which

$$
\mathscr{P}<0 .
$$

We may then choose an initial displacement $(\xi, \eta)$ satisfying (5.16) such that $\mathscr{P}(0)<0$. We also choose the initial velocity $(u, w)$ such that $\mathcal{K}(0)<|\mathcal{P}(0)|$, whence $\mathscr{E}(0)<0$. Now, according to the definition (5.12),

$$
\mathscr{E}_{\lambda}(0)=\mathscr{E}(0)-\lambda \dot{M}(0)+2 \lambda^{2} \mathcal{M}(0) .
$$

For the initial condition with $\mathscr{E}(0)<0, \mathscr{E}_{\lambda}(0)$ is a quadratic polynomial in $\lambda$ with a positive coefficient of $\lambda^{2}$ and a negative constant term. Therefore, it is always possible to find a positive value of $\lambda$ such that $\mathscr{E}_{\lambda}(0)<0$; the interval of admissible values is

$0<\lambda<\Lambda \equiv \frac{\dot{M}(0)}{4 \mathscr{M}(0)}+\left\{\left(\frac{\dot{M}(0)}{4 \mathcal{M}(0)}\right)^{2}-\frac{\mathscr{E}(0)}{2 \mathcal{M}(0)}\right\}^{1 / 2}$.

It is evident from (5.18) that $\Lambda>0$ for any initial condition with $\mathscr{E}(0)<0$, so that the interval is finite. Finally, (5.14) and (5.15) imply that for such an initial condition $\mathscr{E}_{\lambda}(t)$ and $\mathcal{P}(t)$ are always negative and become exponentially more negative with time.

To establish instability it is necessary to demonstrate growth of the disturbance norm $M^{1 / 2}$. This is done by noting that, from the definition (5.7b),

$$
\begin{array}{r}
\mathcal{P}(t) \geqslant \iint_{D} \frac{1}{2}\left\{\left(R_{\xi \xi}-\left|R_{\xi \eta}\right|\right) \xi^{2}+\left(R_{\eta \eta}-\left|R_{\xi \eta}\right|\right) \eta^{2}\right\} \\
\times d x d z,
\end{array}
$$

and hence

$\mathcal{P}(t) \geqslant(-\kappa) \iint_{D} \frac{1}{2}\left(\xi^{2}+\eta^{2}\right) d x d z=-\kappa \mathcal{M}(t)$,

where

$$
\kappa \equiv \sup _{x, z}\left\{\left|R_{\xi \eta}\right|-R_{\xi \xi},\left|R_{\xi \eta}\right|-R_{\eta \eta}\right\} .
$$

Since $P(0)<0$ for the initial condition under consideration and therefore $\kappa>0,(5.20)$ together with (5.15) proves that the disturbance grows according to

$$
M(t)>\frac{\left|\mathscr{E}_{\lambda}(0)\right|}{\kappa} e^{2 \lambda t}, \quad 0<\lambda<\Lambda .
$$

The inequality (5.22) is the major result of this section. It provides an a priori estimate of the growth rate of a symmetric instability in terms of the basic-flow parameters.

We now consider the special case of an initial condition with $\mathscr{P}(0)<0$ and

$$
\begin{aligned}
u(x, z, 0) & =\xi_{t}(x, z, 0)=\lambda \xi(x, z, 0), \\
w(x, z, 0) & =\eta_{t}(x, z, 0)=\lambda \eta(x, z, 0),
\end{aligned}
$$

in which case $\mathscr{K}_{\lambda}(0)=0$ and $\mathscr{E}_{\lambda}(0)=\mathscr{P}_{\lambda}(0)$. From 
(5.12b) it is then clear that the conditions $\lambda>0, \mathscr{E}_{\lambda}(0)$ $=\mathcal{P}_{\lambda}(0)<0$ are satisfied for all $\lambda$ in the interval

$$
0<\lambda<\Lambda^{*} \equiv\left(\frac{|\mathcal{P}(0)|}{\mathcal{M}(0)}\right)^{1 / 2} \text {. }
$$

From $(5.15)$ and that $\mathscr{E}_{\lambda}(0)=\mathscr{P}_{\lambda}(0)$ it follows that

$$
\mathscr{P}(t)<\mathscr{P}_{\lambda}(0) e^{2 \lambda t},
$$

with $\lambda$ chosen within the interval (5.24). Finally, from (5.25)

$$
M(t)>\frac{\left|\mathcal{P}_{\lambda}(0)\right|}{\kappa} e^{2 \lambda t}, \quad 0<\lambda<\Lambda^{*}
$$

This provides, in a certain sense to be made clear, the maximally attainable estimate of disturbance growth.

\section{e. Estimate from above of increase in disturbance amplitude}

From the main inequality (5.14) we may also obtain an estimate from above of the increase in disturbance amplitude. The idea is to find a value of $\lambda$ such that the functional $\mathscr{P}_{\lambda}$ is positive definite for any displacement field $(\xi, \eta)$. In this case the functional $\mathscr{E}_{\lambda}$ is also positive definite, and as a consequence (5.14) provides an upper bound on disturbance growth.

To this end, consider the quantity $\Lambda^{+}$defined by

$$
\Lambda^{+} \equiv \sup \Lambda^{*}
$$

where the supremum is taken over all displacement fields $(\xi, \eta)$ for which $\mathcal{P}(0)<0$. [This extremization problem is mathematically equivalent to that of determining the maximum of the quadratic functional $-\mathcal{P}(0)$ subject to the constraint $M(0) \equiv 1$.] From (5.12b) and the definition of $\Lambda^{*}$ it then follows immediately that $\mathcal{P}_{\lambda}(0)>0$ whenever $\lambda>\Lambda^{+}$. The result applies a fortiori to displacement fields for which $\mathscr{P}(0)$ $>0$. Thus, the functionals $\mathscr{P}_{\lambda}(0)$ and therefore $\mathscr{E}_{\lambda}(0)$ are positive definite for any $\lambda=\Lambda^{+}+\epsilon$ with $\epsilon>0$, in which case (5.14) takes the form

$$
\mathscr{E}_{\Lambda^{++\epsilon}}(t) \leqslant \mathscr{E}_{\Lambda^{++\epsilon}}(0) e^{2\left(\Lambda^{+}+\epsilon\right) t} .
$$

Now, (5.12b) together with the fact that $\mathcal{K}_{\lambda} \geqslant 0$ implies

$$
\left(\Lambda^{+}+\epsilon\right)^{2} M=\mathscr{P}_{\Lambda^{+}+\epsilon}-\mathscr{P} \leqslant \mathscr{E}_{\Lambda^{+}+\epsilon}-\mathscr{P},
$$

whence

$$
\left(\Lambda^{+}+\epsilon\right)^{2} M-\left(\Lambda^{+}\right)^{2} M \leqslant \mathscr{E}_{\Lambda^{+}+\epsilon}-\mathscr{P}_{\Lambda^{+}} \leqslant \mathscr{E}_{\Lambda^{+}+\epsilon}
$$

after using

$$
\mathcal{P}_{\Lambda^{+}} \geqslant 0 \text {. }
$$

Putting (5.30) together with (5.28) then yields

$$
M(t) \leqslant \frac{\mathscr{E}_{\Lambda^{+}+\epsilon}(0)}{\left(2 \epsilon \Lambda^{+}+\epsilon^{2}\right)} e^{2\left(\Lambda^{++\epsilon}\right) t}, \quad \epsilon>0 .
$$

It may be noted that the upper estimate of disturbance growth provided by (5.32) is virtually identical to the lower estimate (5.26) for the most dangerous instability. Thus, we have a very tight estimate of the largest growth rate.

\section{f. Comparison with linear normal-mode analysis}

In a linear normal-mode analysis, we have from (5.1)

$$
u=\sigma \xi, \quad w=\sigma \eta
$$

where $\sigma$ is the growth rate. Multiplying (5.3a) and (5.3b) by $\xi$ and $\eta$, respectively; adding the result; and integrating the sum over the domain yields

$$
\begin{aligned}
& \sigma^{2} \iint_{D}\left(\xi^{2}+\eta^{2}\right) d x d z \\
& =-\iint_{D}\left(R_{\xi \xi} \xi^{2}+2 R_{\xi \eta} \xi \eta+R_{\eta \eta} \eta^{2}\right) d x d z \\
& \Leftrightarrow \sigma^{2}=\frac{(-\mathcal{P}(0))}{M(0)} .
\end{aligned}
$$

Therefore, the growth rate given by the normal-mode analysis is equal to the greatest lower bound estimated by (5.24) and (5.26). Since the least upper bound from (5.32) is given by the same expression, these estimates give the exact growth rate of the normal mode (if a normal mode exists).

\section{g. Further considerations}

The linear analysis of this section is considerably more general than the normal-mode analysis, as can be seen from the following example.

Suppose our domain $D$ is divided into two subdomains, $D_{+}$and $D_{-}$, where the potential vorticity is initially positive in $D_{+}$and negative in $D_{-}$. Normal-mode analysis can tell us nothing about stability in such a situation. In contrast, the lower bound estimated for the growth rate shows that as long as $\Lambda$ given by (5.18) is positive, then the perturbation will grow in the sense that $\mathcal{M}(t)$ increases with time. In particular, if one takes $u(x, z, 0)=0=w(x, z, 0)$, then growth will occur if

$$
\mathscr{P}_{D_{-}}(0)<0, \quad\left|\mathscr{P}_{D_{-}}(0)\right|>\mathscr{P}_{D_{+}}(0) .
$$

On the other hand, the upper bound estimate shows that growth will not occur if $\mathcal{P}(0)$ is non-negative.

\section{Instability of a moist atmosphere}

In this section we consider the problem of a moist atmosphere with saturated ascent and unsaturated descent. This system is much more difficult to analyze than the moist adiabatic system that has been considered earlier, and general stability theorems of the type derived in sections 2 and 3 do not seem to be possible. 
For the instability problem, however, progress is possible in the case of nonoscillatory disturbances (as is the case with symmetric instabilities), since then the regions of ascent and descent are fixed in space. The regions are not known a priori, of course, but the point is that for any given disturbance they do not change in time.

Thus, we assume that the domain $D$ is divided into two subdomains, $D_{>}$and $D_{<}$(which are determined in any given realization by the initial conditions), wherein $w>0$ and $w<0$, respectively. The full set of governing equations is the same as (2.2), with the one exception that $(2.2 \mathrm{e})$ is replaced by

$$
\frac{D \theta}{D t}=\left\{\begin{array}{lll}
0, & \text { in } D_{<} \\
k w, & \text { in } D_{>},
\end{array}\right.
$$

with $k>0$ some constant parameter. As before, our interest is in the stability of disturbances to some equilibrium state (2.13). The linearized form of (6.1) about such a state may be written

$$
\theta_{t}^{\prime}+u \hat{\Theta}_{x}+w \hat{\Theta}_{z}=0
$$

where

$$
\hat{\Theta} \equiv\left\{\begin{array}{lll}
\Theta, & \text { in } & D_{<} \\
\Theta-k z, & \text { in } & D_{>} .
\end{array}\right.
$$

It follows that $(5.2 \mathrm{a})$ is replaced with

$$
\theta_{t}^{\prime}=-\xi \hat{\Theta}_{x}-\eta \hat{\Theta}_{z},
$$

while (5.2b) is unchanged, whence (5.3a) and (5.3b) apply provided we take

$$
\left(\begin{array}{ll}
R_{\xi \xi} & R_{\xi \eta} \\
R_{\eta \xi} & R_{\eta \eta}
\end{array}\right) \equiv\left(\begin{array}{ll}
f M_{x} & f M_{z} \\
\frac{g}{\theta_{0}} \hat{\Theta}_{x} & \frac{g}{\theta_{0}} \hat{\Theta}_{z}
\end{array}\right)
$$

instead of (5.4). The remainder of the analysis of section 5 then follows without alteration. The only difference arises in the optimization problem (5.27) in that different displacement fields $(\xi, \eta)$ imply different definitions of the regions $D_{<}$and $D_{>}$and, hence, different equilibrium fields $\hat{\Theta}$. A detailed exploration of the technical implications of this interesting optimization problem is beyond the scope of the present paper.

\section{Discussion}

Most of the well-known sufficient criteria for hydrodynamical stability have been derived in the first instance under the restrictive assumption of normalmode disturbances. It was shown by Fjørtoft (1950), however, that many of these criteria, such as Rayleigh's inflection-point and centrifugal-stability criteria, can also be derived under more general conditions from the global invariants of the problem. It thus turns out that in many cases of interest there is a fundamental link between symmetries, conservation laws, and stability theorems, a fact that tends to be obscured by the normal-mode framework. This link also means that such stability theorems can often be extended to finite amplitude, a point that was apparently first recognized by Arnol'd (1965, 1966). For more discussion of this subject, one may refer to the articles by Holm et al. (1985) and McIntyre and Shepherd (1987), among others.

To this point, the most successful applications of the Fjørtoft-Arnol'd stability method in geophysical fluid dynamics have been to barotropic and baroclinic quasigeostrophic flow, to stratified equilibria, and to axisymmetric homogeneous flow. A major purpose of the present paper has been to point out that the wellknown sufficient criterion for symmetric stability, namely static stability together with positivity of $f$ times the potential vorticity (Fjortoft 1950; Stone 1966; Hoskins 1974), is also derivable from this approach and that a nonlinear stability theorem follows directly. An extension of the stability theorem to moist adiabatic (saturated) systems is also possible.

Nonlinear stability is an extremely powerful result. Even flows that are unstable may be regarded as finiteamplitude disturbances to stable flows, and if such stable flows are sufficiently nearby (in a mathematically precise sense), then one may use the relevant nonlinear stability theorem to provide rigorous upper bounds on the nonlinear saturation of disturbances to the unstable flow (Shepherd 1988a). This method is developed in the present context for symmetric instability and applied to the special but important case of a symmetrically unstable baroclinic flow $V(z) \propto z$. The resulting bounds constrain the kinetic energy (in the crossstream plane) of the growing disturbance. In that sense they represent a rigorous upper bound on the available energy in the initial unstable flow. The best (i.e., most constraining) bound would obtain on determining the minimum such bound over all possible choices of stable basic flows. In practice this minimization calculation can become technically complicated; in this paper only a few simple estimates have been provided, which could doubtless be improved upon.

The notion of treating the unstable flow as a finiteamplitude disturbance to a stable flow and determining the pseudoenergy associated with this disturbancewhich then provides a bound on the available energy for the cross-stream flow-is precisely analogous to the usual determination of the available potential energy (APE) associated with departures from a resting, stably stratified basic state, which is likewise derivable from the pseudoenergy using Casimir invariants (Shepherd 1993). Indeed, in his classic paper on APE, Lorenz (1955) suggested that for nonresting basic states that were stable, one might wish to replace the APE with 
some other quantity that would reflect the dynamical stability constraints. In general, this is a nontrivial matter, but for symmetric flows the generalization is obvious: one simply needs to include the centrifugal potential energy together with the gravitational energy and derive an available energy using the pseudoenergy. It is just such a construction that has been made here to obtain the rigorous saturation bounds. Of course, as Lorenz (1955) presciently pointed out, the resulting available energy is a functional of the background flow in question.

It is important to appreciate the distinction between the sort of saturation bounds derived from the nonlinear stability theorem and parcel-based estimates of the available potential energy that can be converted to kinetic energy in slantwise convection (Emanuel 1983). The parcel calculations are physically well motivated but suffer from the lack of rigour inherent in any parcel method (Thorpe et al. 1989), as well as a sensitivity to the angle at which the presumed parcel motion takes place: compare, for example, (4.31) with (4.33). On the other hand, the rigorous upper bounds are just that, upper bounds, and may not provide particularly good estimates of the amplitudes actually obtained in a real instability. In that sense, the two kinds of theoretical calculations are quite complementary.

The present analysis might be criticized for assuming inviscid dynamics. Indeed, there is something inherently unsatisfying about inviscid analyses of nonlinear symmetric instability, for it is well known (Thorpe and Rotunno 1989) that no equilibration of symmetric instability is possible without viscosity. Although the underlying stability theory is based on a Hamiltonian formulation of the dynamics, this does not necessarily preclude the possibility of obtaining saturation bounds in a forced-dissipative context (cf. Shepherd 1988a, section 4). It is difficult, however, to see how conventional formulations of viscosity could be included selfconsistently in the present context, as then the background state would be time-dependent.

Acknowledgments. HRC and TGS wish to acknowledge support from the Natural Sciences and Engineering Research Council and the Atmospheric Environment Service of Canada. This work was made possible during a visit by VAV to the Physics Department at the University of Toronto, which was supported in part by the Department's Blyth Fund. The constructive comments of the referees were much appreciated.

\section{REFERENCES}

Arnol'd, V. I., 1965: Conditions for nonlinear stability of stationary plane curvilinear flows of an ideal fluid. Dokl. Akad. Nauk. SSSR, 162, 975-978. [English translation: Sov. Math., 6, 773777.]

$\ldots, 1966$ : On an a priori estimate in the theory of hydrodynamical stability. Izv. Vyssh. Uchebn. Zaved. Matematika, 54, 5, 3-5. [English transl.: Amer. Math. Soc. Transl., Ser. 2, 79, 267-269.]

Benjamin, T. B., 1984: Impulse, flow force and variational principles. IMA J. Appl. Math., 32, 3-68.

, 1986: On the Boussinesq model for two-dimensional wave motions in heterogeneous fluids. J. Fluid Mech., 165, 445-474.

Bennetts, D. A., and B. J. Hoskins, 1979: Conditional symmetric instability-A possible explanation for frontal rainbands. Quart. J. Roy. Meteor. Soc., 105, 945-962.

Chandrasekhar, S., 1970: Ellipsoidal Figures of Equilibrium. Yale University Press, 288 pp.

Charney, J. G., 1973: Planetary fluid dynamics. Dynamic Meteorology, P. Morel, Ed., Reidel, 97-351.

Chetaev, N. G., 1955: Stability of Motion. (in Russian). GITTL, $207 \mathrm{pp}$.

Emanuel, K. A., 1983: The Lagrangian parcel dynamics of moist symmetric instability. J. Atmos. Sci., 40, 2368-2376.

Fjørtoft, R., 1950: Application of integral theorems in deriving criteria of stability for laminar flows and for the baroclinic circular vortex. Geofys. Publ., 17(6), 1-52.

Holm, D. D., J. E. Marsden, T. Ratiu, and A. Weinstein, 1985: Nonlinear stability of fluid and plasma equilibria. Phys. Rep., 123, $1-116$.

Hoskins, B. J., 1974: The role of potential vorticity in symmetric stability and instability. Quart. J. Roy. Meteor. Soc., 100, 480482.

Lorenz, E. N., 1955: Available potential energy and the maintenance of the general circulation. Tellus, 7, 157-167.

McIntyre, M. E., and T. G. Shepherd, 1987: An exact local conservation theorem for finite-amplitude disturbances to non-parallel shear flows, with remarks on Hamiltonian structure and on Arnol'd's stability theorems. J. Fluid Mech., 181, 527-565.

Moiseev, N. N., and V. V. Rumyantsev, 1965: Dynamics of a Body with Cavities Containing a Liquid. (in Russian). Nauka, 439 pp.

Ooyama, K., 1966: On the stability of the baroclinic circular vortex: A sufficient criterion for instability. J. Atmos. Sci., 23, 43-53.

Ripa, P., 1983: General stability conditions for zonal flows in a onelayer model on the $\beta$-plane or the sphere. J. Fluid Mech., 126, 463-489.

Salmon, R., 1988: Hamiltonian fluid mechanics. Ann. Rev. Fluid Mech., 20, 225-256.

Shepherd, T. G., 1988a: Rigorous bounds on the nonlinear saturation of instabilities to parallel shear flows. J. Fluid Mech., 196, 291322.

1988b: Nonlinear saturation of baroclinic instability. Part I: The two-layer model. J. Atmos. Sci., 45, 2014-2025.

, 1989: Nonlinear saturation of baroclinic instability. Part II: Continuously stratified fluid. J. Atmos. Sci., 46, 888-907.

- 1990: Symmetries, conservation laws, and Hamiltonian structure in geophysical fluid dynamics. Advances in Geophysics, Vol. 32, Academic Press, 287-338.

- 1991: Nonlinear stability and the saturation of instabilities to axisymmetric vortices. Eur. J. Mech. B/Fluids, 10, 93-98.

- 1993: A unified theory of available potential energy. Atmos.Ocean, in press.

Stone, P. H., 1966: On non-geostrophic baroclinic instability. J. Atmos. Sci., 23, 390-400.

Szeri, A., and P. Holmes, 1988: Nonlinear stability of axisymmetric swirling flows. Phil. Trans. Roy. Soc. London A, 326, 327-354.

Thorpe, A. J., and R. Rotunno, 1989: Nonlinear aspects of symmetric instability. J. Atmos. Sci., 46, 1285-1299.

- B. J. Hoskins, and V. Innocentini, 1989: The parcel method in a baroclinic atmosphere. J. Atmos. Sci., 46, 1274-1284.

Vladimirov, V. A., 1985: Variational principle and a priori estimate of stability for stratified fluid. Dynamics of Continuous Media (in Russian), 72, 12-18.

, 1986a: Analogues of the Lagrange theorem in the hydrodynamics of whirling and stratified liquids. Prikl. Mat. Mekh., 50, 
5, 727-733. (English transl.: J. Appl. Math. Mech., 50(5), 559564.)

$-1986 \mathrm{~b}$ : Conditions for nonlinear stability of flows of an ideal incompressible liquid. Zhurn. Prikl. Mekh. Tekh. Fiz., 3, 7078. [English transl.: J. Appl. Mech. Tech. Phys., 27, 3, 382389.]

_ 1987: Integrals of two-dimensional motions of a perfect incompressible fluid of nonuniform density. Izv. Akad. Nauk. SSSR, Mekh. Zhid. Gaza, No. 3, 16-20. [English transl.: Fluid Dyn., 22(3), 340-343.]
- 1989: Instability of the equilibrium of fluids. Zhurn. Prikl. Mekh. Tekh. Fiz., No. 2, 108-116. (English transl.: J. Appl. Mech. Tech. Phys., 30(2), 269-276.)

- 1990: Direct Lyapunov's method in problems of fluid equilibrium instability. Arch. Mech. (Warsaw), 42(4/5), 376-388.

- , and V. V. Rumyantsev, 1990: On inversion of Lagrange theorem for a solid body with a cavity containing viscous liquid. Prikl. Mat. Mekh., 54(2), 303-310. [English transl.: J. Appl. Math. Mech., 54.] 